\title{
A general-purpose facility for adaptive testing in psychoacoustics
}

\author{
MARK YAPHE \\ University of Waterloo, Waterloo, Ontario, Canada \\ and \\ EMMET RAFTERY and DONALD G. JAMIESON \\ University of Western Ontario, London, Canada
}

\begin{abstract}
We describe a general-purpose, programmable system that provides high-quality, low-cost devices for experimentation in psychoacoustics and speech perception. The system is controlled by a host computer (e.g., an IBM PC), over a serial line. Through the use of a high-level, general-purpose experiment control program, the designed interconnection of devices can be specified logically, and the settings of the devices modified dynamically, during the experiment.
\end{abstract}

While the stimulus and experimental-control requirements for speech and hearing research are demanding, they are relatively uniform across experiments and laboratories. One must first specify and control the spectra and levels of the presented stimuli, including the contribution of the overall frequency response of the complete electroacoustic environment to the level of the acoustic coupling to the subject's ear. To ensure that testing occurs under standardized conditions, equipment must be calibrated at the beginning of each experimental session. Then, one must be able to present the desired signals in the sequence and at the times specified.

A relatively large number of special-purpose and/or expensive components are required for such research: specialized equipment is typically required to filter (antialias) signals for digital-to-analog conversion, to amplify and/or attenuate signals, and to mix signals from different sources. Typically, the preparation for an experiment involves the manual interconnection of the devices used during the experiment, with the associated delays, as well as opportunities for human error. Moreover, the interconnection of equipment with different grounding and cabling systems tends to introduce noise (e.g., $60 \mathrm{~Hz})$ into the circuitry. ${ }^{1}$ Also, when two or more experimental conditions requiring different configurations of equipment are used during a given testing session, such concerns are multiplied.

In order to improve the efficiency with which experiments can be set up and controlled while reducing the possibilities for noise and error, researchers have adopted several approaches. The first is to use a connector panel,

This work was supported, in part, by grants from the Natural Sciences and Engineering Research Council, and from Health and Welfare Canada, to Donald G. Jamieson. Correspondence should be addressed to Donald G. Jamieson, Speech Communication Laboratory, Department of Communicative Disorders, University of Western Ontario, Elborn College, London, Ontario N6G 1H1, Canada. in which the inputs and outputs for each device are permanently wired to a bank of connectors (e.g., phono jacks); when two devices are to be interconnected, the researcher makes the connection directly on this panel, with the appropriate cable.

An improved but more costly approach is to use removable plugboard panels (e.g., the MacPanel). Such systems use a two-part panel-one portion onto which devices are permanently connected, together with a second, removable board portion on which connections can be made. When the removable board is in place, the two panels form a "sandwich," with the devices interconnected by the wires on the plugboard. To switch to another configuration of devices, one removes the one panel and replaces it with another one wired for the other configuration.

A natural step toward improving the efficiency with which devices can be interconnected would involve integrated hardware, with a logical switching system that would permit the devices to be interconnected under computer control. This approach would have several advantages. First, the system could be configured in the desired fashion quickly, by means of simple software commands. Second, the possibilities for noise and error would be minimized. Third, the approach would permit a variety of speech and auditory perceptual experiments, requiring different equipment configurations, to be used within a single experimental session without noticeable interruption for the subject. Finally, such a system would be particularly well-suited for adaptive testing, in which the sequence of experimental testing conditions depends on the individual listener's responses from trial to trial (see Levitt, 1971). Such adaptive testing is desirable because it allows psychoacoustic abilities to be measured in a particularly efficient fashion, but the required level of control over the test parameters does introduce an additional level of complexity for instrumentation and software.

The present paper describes a system that provides highquality, low-cost devices for experimentation in psycho- 
acoustics and speech perception. The system comprises a general-purpose controller and several digitally programmable analog "building blocks." This system has been designed to supersede previous systems based on generalpurpose controllers, which are more limited in the number and type of devices to be controlled, do not provide an ergonomic user interface, and/or require more supporting hardware (Dillon, Millman, Tombaugh, Ferguson, \& Bezanson, 1979; Palya, Ortega, \& Wilson, 1982).

With the present approach, experiments are controlled by a host computer (such as an IBM AT-compatible), using a high-level language. During an experiment, the desired settings for each device are transmitted to the controller through the RS-232 port of the host computer, by means of high-level commands. The hardware design and the host-software interface are described below, from both the user's and the programmer's perspectives.

\section{Overview of System Hardware}

Our system consists of a general-purpose controller, together with several programmable analog devices, enclosed in a single rack-mountable box. The analog devices include programmable gain controls, antialiasing (lowpass) filters, multiplexors, and power amplifiers. These analog devices are connected to the expansion (I/O) bus of the controller's processor by means of plug-in cards, in a manner similar to that for the familiar peripheral cards for the IBM PC. The analog devices are well-shielded from the digital hardware and buses of the controller, to reduce noise in the analog circuitry. Multiple copies of any of the analog cards may be installed on the expansion bus; the addresses of each card are switch-selectable.

Communication with the controller. Communication with the controller occurs either manually, with pushbuttons, or from a host computer, through a standard serial interface (RS-232). The RS-232 interface was chosen because it is available on most standard personal computers, so that it does not restrict the type of host computer. The serial port is configured as Data Communications Equipment (DCE), and can be configured to support one of several popular handshaking protocols, including XON/XOFF, RTS/CTS, and variations thereof.

The second user interface occurs with a set of pushbuttons, together with an LCD display. The pushbuttons are used to select the desired "softkey": at any given time, the LCDs display, in menu fashion, the function of each of the pushbuttons. The current version of the controller software does not provide strong support for the LCD interface.

The general-purpose controller. The general-purpose controller is a microprocessor-based circuit, external to the host microcomputer, which controls several programmable analog devices. The INTEL 8096BH microcontroller was selected as the CPU for the system. Its fast $(6-\mathrm{MHz})$ execution speed, on-board timers, and internal full duplex serial port were characteristics that made it a suitable choice for the CPU.

The controller's memory subsystem comprises four elements: two programmable ROM chips (EPROMs), one electrically erasable, programmable ROM chip (EEPROM), and one RAM chip. The first of the two EPROM chips contains the software for the 8096BH processor. This chip includes routines to communicate with host processors over the serial port, to control the LCD display and the push-button inputs, to interpret commands from the host, and to configure the analog devices. The second EPROM chip contains data tables that describe the possible device types and characteristics, together with tables containing sine and cosine values for use in calculations.

The EEPROM chip is a type of memory that can be read from or written to. Unlike RAM chips, it retains its memory even when the machine is powered off. Hence, this chip is used to store information about the system's operating setup, including details about handshaking protocol for the serial port and configurations of the analog devices. Finally, the RAM chip is used for storage of data and variables while the 8096 is executing programs.

The expansion I/O bus. The expansion I/O bus is simply a buffered subset of the 8096's buses. This bus includes all the necessary read/write timing signals, the data bus, and the least significant 12 bits of the address bus. That is, the expansion I/O memory occupies the top $4 \mathrm{~K}(4,096$ bytes $)$ of the processor's memory space. The most significant four bits of the address are decoded for the analog boards by the controller board.

Each of the analog cards connected to the expansion I/O bus has an associated range of addresses. A DIP switch on each analog board selects the address range at which the card will be accessed. Thus, multiple copies of a particular card type may be installed in the system, each set to a different address. This approach differs from that taken in a series of recent reports on the Walter/Palya controller (D'Andrea \& Knepton, 1988; Palya, 1988; Walter \& Palya, 1984). In that system, devices are controlled through the manipulation of a limited number of separate control lines, which necessitate the addition of subsequent controllers when these lines have been exhausted. Moreover, to allow the Walter/Palya controller to send data bytes to modify the settings of instruments would require the use of eight of the controller's control lines at a time ( 8 bits of information per data byte), thus depleting the number of control lines quite rapidly. The controller in the present system has the capability of addressing 4,096 bytes, so that a full set of analog instruments can be controlled.

Analog 1/O. All analog I/O (of the analog cards) occurs through terminal connectors on the rear of the cards. Each analog input and each output supports both differential and single-ended signal types, to provide flexibility in connections to devices that are external to the box. Interconnections between cards are made with these terminals on the cards.

Interconnections to external devices are made by wiring the terminals on individual cards to the appropriate connectors on the (hinged) rear of the box. XLR, phone, phono, and BNC connectors are used to eliminate much of the need for custom cabling. More importantly, this 
approach allows differential signals from external devices to be connected to differential I/O circuitry on the analog cards. Similarly, single-ended external devices may be connected to single-ended analog circuitry on the cards, significantly reducing the opportunities for the noise and grounding problems that often occur with the custom cabling and patch-panel approaches. The connections between external devices and the analog circuitry, described in ensuing sections of this paper, are therefore made just once, when the hardware is initially configured. They are not changed from experiment to experiment.

Analog multiplexor boards. An analog multiplexor acts as a programmable switch, allowing any one of the various inputs to be connected to its output. Each multiplexor board in the system has two independent analog switches, selecting one of four possible inputs. Since the level of the input signal (from an external device) is not necessarily at the level of the device to which the signal is routed, outputs of the switches are fed into programmable gain blocks. These gain blocks allow the signals to be amplified/attenuated from $+60 \mathrm{~dB}$ to $-85 \mathrm{~dB}$ in steps of $1.5 \mathrm{~dB}$.

In some situations, the mixing of two signals is required. Hence, an adder circuit is fed by the gain-adjusted output signals from both multiplexors. This output is also available on the rear of the cards.

Antialiasing filters. To prevent distortion due to aliasing associated with $\mathrm{A} / \mathrm{D}$ and $\mathrm{D} / \mathrm{A}$ conversion, the frequency spectrum of signals going into or out of a digital signal processing (DSP) system must be band-limited to allow only frequencies below one half of the digital sampling frequency. Because a variety of sample frequencies are used in different applications, antialiasing filters of correspondingly varied frequencies are required. As a consequence, a set of programmable antialiasing filters was designed for the project. With this system, a single card can be programmed to cut off at one of four possible frequencies. The filters are 10th-order Chebychev filters, with $0.1 \mathrm{~dB}$ of ripple in the pass-band and rolloff at $>60 \mathrm{~dB} /$ octave. The cutoff frequencies for the filters can be customized at the time of manufacture, depending on the requirements of the user. The general-purpose controller can determine the frequencies that are available on a filter card by reading the values stored on the EPROM of the filter card.

Power-amplifier boards. The power-amplifier boards of the system are designed to drive standard output transducers such as loudspeakers or headphones. Two independent power amplifiers are included on each board. Each power amplifier can deliver $5 \mathrm{~W}$ of power (RMS) to an 8- $\Omega$ load, with $0.01 \%$ total harmonic distortion at its rated power. Each amplifier's gain can be varied (programmably) by $85 \mathrm{~dB}$, in $1.5-\mathrm{dB}$ steps. The amplifier can also be programmed to use an (optional) external potentiometer as the volume control. The upper limits of gain (and sensitivity) of the manual volume control can be programmably specified.

Several protection circuits have been built into the amplifier circuitry. The amplifiers will protect themselves from damage due to overcurrent on their outputs (due to short circuits on output, or to too many loads connected) by shutting off their output circuitry for a few seconds, and informing the general-purpose controller. This allows the controller to take corrective action, and to inform the host processor of the occurrence. A second protection circuit has been included to protect the external circuitry and/or the test subject. The amplifiers can be programmed to inform the controller if the output voltage exceeds a preprogrammed value. The controller can then take corrective action and/or inform the host processor of the occurrence.

\section{A Configuration Example}

Figure 1 displays an example of a system that contains a simple configuration of analog components. In this example, several analog devices are connected to the inputs of an analog multiplexor-a tape playback device, a signal generator, a noise generator, and the output of a microphone preamplifier. The output of this multiplexor is connected to the input of a programmable antialiasing filter, the output of which is connected to one of the A/D inputs of an Ariel DSP-16 system. Hence, the DSP-16 may be used to digitize the stimuli from any of the sources to the first multiplexor. The output of the DSP-16 is routed through a second programmable antialiasing filter, to a second analog multiplexor. Also connected to the second multiplexor are the tape playback device and signal generator. The output of this multiplexor is connected to a programmable-gain power amplifier, and to an output transducer. With the configuration in Figure 1, the DSP16 board can be used to output previously digitized signals stored on the host computer's disks, or to implement a DSP algorithm on the stored signals and then output them. In fact, because of the flexibility of the system, any of the inputs to the first multiplexor can be digitally filtered in real time, and sent to the output transducer. The system can also be used to directly output stimuli from the tape playback device or the signal generator. Moreover, since the complete signal path can be changed at any time by the host processor, without any need for manual rewiring, a subject can be tested under two or more experimental conditions, requiring different equipment configurations, without any delay in moving between conditions.

\section{Host-Command Interface}

In the current version of the controller's software, responsibility for all aspects of program control rests with the host computer. That is, the controller currently supports just two basic host commands: store a byte of data into, or read a byte value from, a memory location or $\mathrm{I} / \mathrm{O}$ port (henceforth referred to as the store and load commands, respectively).

The store and load commands use a simple protocol. When the system has finished processing a command from the host, it returns the execution status of the command (passed or failed, together with reason for failure). After a load command, the controller returns the ASCII equiva- 


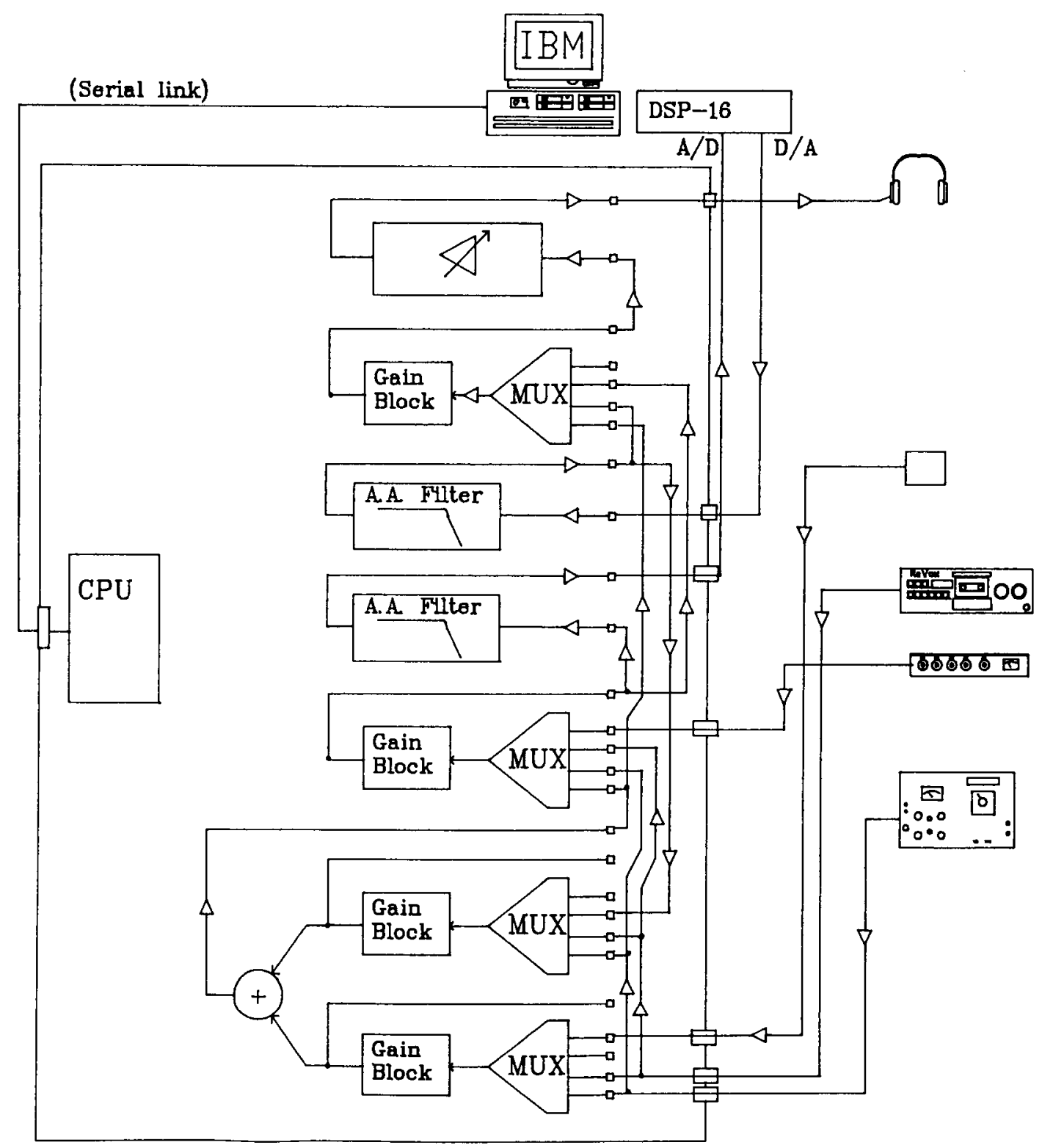

Figure 1. An example of the controller configured for a speech-perception experiment involving taped speech mixed with noise. The IBM AT configures the system through an RS-232 connection to the controller. The system configuration and the settings of individual system components can be altered during an experiment.

lent of the byte in the requested memory location, followed by a status byte. To minimize the idling time of both processors (host and controller), the system can buffer a limited number of commands.

However, due to the restrictive nature of the initial version of the controller software, the user must decompose more elaborate functions into a sequence of store and load commands, which are sent, one by one, to the system. The analog devices are controlled by sending store commands to the appropriate addresses on the analog boards. The store command contains the hex memory location as- sociated with the device to be controlled, and the hex value to be sent to the device. Command strings and responses (to and from the controller) are delimited by carriage return and line feed (CR; LF) sequences.

With the current version of software, the system can be configured for a given experiment or application by one of two methods: with the disk operating system of the host computer (DOS), or dynamically with the experimental control program. With DOS, one sends the host commands to the general-purpose controller by copying a file of commands to the COM (serial) port, or by 
using the ECHO command (on the IBM PC) to send individual commands. An example of how a command would be sent to the controller from an IBM host is:

$$
\text { ECHO S < destination_addr > < value > COM1: }
$$

where <destination_addr > is the four-digit hexadecimal address of the device to be controlled, and $<$ value $>$ is a two-digit hexadecimal value (byte) to be stored in that address.

Dynamically from the experimental control program, an identical string would be sent to the controller. However, a high-level language, such as Pascal, that allows string assignments to variables, greatly simplifies the building of the command strings. For example, a string can be constructed as:

$$
\text { 'S',Mux__1,' ',Tape_1 }
$$

where Mux_1 is a preassigned string constant, which is the address of one of the multiplexors in the system, and Tape__ 1 is a two-digit string constant to be written to the multiplexor card, which would cause it to select the input to which the tape playback device was connected. Future versions of the controller's software will simplify this procedure.

\section{User's Perspective}

The most important task associated with setting up the present system hardware is to determine the hardware configuration for which all the desired experiments can be performed without manual rewiring. Once the appropriate analog cards have been selected to configure the hardware setup, the addresses of the individual cards must be selected (with the DIP switches on the cards) so that the address space does not overlap for any two cards. A simple program for the IBM PC has been developed to assist in address selection and documentation.

The analog cards are installed by sliding them into any of the 10 card-edge connectors in the card cage of the box. All internal wiring (from card to card) is done by means of the differential I/O terminals on the cards. The external I/O connections are made to appropriate connectors on the hinged back panel of the box. Care must be taken that the input wiring connects differential signals to differential inputs on the cards, and that single-ended signals (especially if delivered over a coaxial cable) are connected to single-ended terminations on the analog boards. Once this wiring is complete, the system can be turned on.

When turned on, the general-purpose controller performs a self-test of all its memory and digital $\mathrm{I} / \mathrm{O}$ devices. When the self-test is complete, a status message will appear on the LCD display, indicating that the system is ready, or that a particular type of failure has occurred. If there are no failures, the system is ready for host commands, and can be operated using the methods outlined in the Host-Command Interface section of this paper.

\section{Sample Application}

One way we have used this system has been to measure speech intelligibility for individual listeners, at various signal-to-noise ratios. Our stimuli are a combination of words stored on disk, and noise produced by a random generator. These signals are preamplified, mixed, filtered, and amplified for delivery to the listener's ear through an insert earphone (ER3A) at the desired level and at the desired signal-to-noise ratio. Signal levels are confirmed with the use of a probe-tube microphone located within the listener's ear canal.

To undertake this experiment, the output of the probetube measurement system is amplified and directed to an antialiasing filter, and then to the A/D input of a DSP-16 board in the host microcomputer. This procedure allows signal levels to be measured and calibrated, through adjustment of the level of the programmable gain control in the system. To measure the signal level, a known signal is output over the D/A converter of the DSP-16 (with the noise switched out). On the basis of the measured response to these signals, the host modifies the settings of the analog components until the measured signal reaches the desired level. The level of the noise channel is calibrated in a similar fashion. Note that the accuracy (resolution) of the A/D channel can be adjusted dynamically, through variation of the gain of the microphone's preamp (in the multiplexor circuit); in this way, the system can use the full resolution of the A/D converter.

The subject's responses are recorded directly by the host PC, by means of either a mouse-graphics interface or pushbutton input, so that the experimental situation can easily be varied adaptively: when a new hardware configuration is required, the system can be reconfigured by software-for example, the attenuation (gain) levels or DSP filters can be modified dynamically.

\section{Future Developments}

Although the hardware aspects of our system are now quite complete, several improvements are planned. One objective is to improve the user interface. Since researchers and programmers currently must understand more of the internal details of the system than seems desirable, future software revisions will focus on the development of the interface for the user. In particular, we will be developing a command parser for the general-purpose controller, and a configuration program for the host processor. With the improved software, the user will be able to specify an eight-character name for each input and output device, at the time the system is initially configured. The configuration software will then download this information to the general-purpose controller, which in turn will store all the configuration information in its EEPROM. Subsequently, each device will be specified by its assigned name, through the serial port, or on the LCD display. When initially turned on, the controller will verify the existence of all peripherals and set them to some specified default configuration. 


\section{REFERENCES}

D'Andrea, J. A., \& KNEPTON, J. (1988). Construction and implementation of a low-cost electronic equipment control interface. Behavior Research Methods, Instruments, \& Computers, 20, 97-99.

Dillon, R. F., Millman, B., Tombaugh, J. W., Ferguson, W. R., \& BEZANson, W. R. (1979). A microcomputer-controlled laboratory: Hardware. Behavior Research Methods \& Instrumentation, 11, 293-300.

LEviTt, H. (1971). Transformed up-down methods in psychoacoustics. Journal of the Acoustical Society of America, 49, 467-477.

PALYA, W. L. (1988). An introduction to the Walter/Palya controller and ECBASIC. Behavior Research Methods, Instruments, \& Computers, 20, 81-87.

Palya, W. L., Ortega, J., \& Wilson, J. B. (1982). An inexpensive
CRT terminal controller/computer. Behavior Research Methods \& Instrumentation, 14, 92-98.

Walter, D. E., \& Palya, W. L. (1984). An inexpensive experiment controller for stand-alone applications or distributed processing networks. Behavior Research Methods, Instruments, \& Computers, 16, 125-134.

\section{NOTE}

1. For example, in our laboratory, one third of the equipment is balanced, using XLR connectors and shielded three-wire cable, one third is single-ended (using Coaxial interconnections), and one third uses one of various "isolated" or other schemes. It is quite difficult for even an experienced electrical engineer to remove all the grounding problems from such a system, especially if the system is to have a "variable configuration."' 